\title{
Hydatidiform mole
}

INSERM

\section{Source}

INSERM. (1999). Orphanet: an online rare disease and orphan drug data base.

Hydatidiform mole. ORPHA:99927

A hydatidiform mole is a benign gestational trophoblastic disease developing during pregnancy. Resulting from an abnormal fertilization characterized by trophoblastic proliferation, normal embryo development is rendered impossible. Hydatidiform moles can be either complete or partial. 